\title{
TWITTER ET LES RELATIONS DE SÉDUCTION ENTRE DÉPUTÉS ET JOURNALISTES
}

\author{
La salle des Quatre Colonnes \\ à l'ère des sociabilités numériques
}

Jonathan CHIBOIS 
'entrée de Twitter à l'Assemblée nationale française ne s'est pas faite sans remous ${ }^{1}$. Au cours du premier semestre de l'année 2010, à plusieurs reprises et pour leur propre bénéfice médiatique, des députés ont utilisé les courts messages à forte audience que sont les tweets pour lever le voile sur quelques nouvelles faisant 1'événement au Palais Bourbon, à la grande satisfaction du monde journalistique ${ }^{2}$. Ces épisodes ont amené en juillet 2010 le Bureau de l'Assemblée ${ }^{3}$ à réfléchir à la meilleure manière d'encadrer l'usage du tweet dans l'enceinte du Palais Bourbon, ainsi que Bernard Accoyer, alors président de l'Assemblée, à déclarer que « les nouvelles technologies sont là, il faut s'en féliciter, elles font circuler l'information de manière de plus en plus rapide. Mais elles ne peuvent remettre en cause le respect du huis clos lorsque l'Assemblée ou une commission décide de siéger à huis clos $\gg{ }^{4}$. Ces mots sont représentatifs du désarroi d'un grand nombre de parlementaires face à ce qui est apparu comme une remise en cause de certains principes de fonctionnement de l'institution par des usages médiatiques émergents. La question du régime de visibilité entourant les affaires publiques traitées à l'Assemblée a fait l'objet de discussions, et la question du huis clos n'en était qu'un aspect. D'une manière plus large, c'est le vieux débat sur la publicisation des activités parlementaires qui ressurgit ici, et avec lui l'épineuse question de la relation des députés et de l'Assemblée avec la presse.

L'histoire des relations entre la presse et le Parlement en France se décompose en trois périodes. Depuis l'Assemblée constituante de 1789, la publicité des activités du Parlement, et en particulier le compte rendu des débats, est assurée de manière volontaire par les journaux de presse, qui commentent et critiquent l'actualité politique pour leur lectorat. Il faudra attendre 1852 pour

1. Je remercie ici les députés, collaborateurs et journalistes qui m'ont confié leur témoignage pour l'élaboration de ce texte et, pour quelques-uns d'entre eux, qui se sont proposés pour une relecture critique. Je remercie également les relecteurs anonymes de la revue pour leurs remarques avisées.

2. L'histoire et les enjeux initiaux de l'adoption de Twitter par les députés sont détaillés dans Chibois (2014).

3. Le Bureau de l'Assemblée est la plus haute autorité collégiale de l'institution.

4. Propos cités par l'AFP, le 2 juillet 2010. 
que l'administration parlementaire publie un compte rendu unique et officiel des débats, de façon à remédier à la pluralité des comptes rendus rédigés par les journaux, nécessairement partisans (Lavoinne, 1999). Jusque-là, comme l'explique Virginie Le Torrec (2005), la liberté de la presse est source d'une continuelle suspicion de la part des pouvoirs publics des régimes politiques successifs qui tentent d'asseoir leur légitimité, les contenus publiés étant à ce point sensibles qu'ils font l'objet de nombreuses dispositions réglementaires, dont des délits passibles d'amende explicitement relatifs au compte rendu des débats. Les années 1880, qui voient s'installer le régime des journalistes parlementaires, ouvrent une seconde période en stabilisant pour la première fois les relations entre la presse et le Parlement. Intégrés à l'institution avec un statut propre, les journalistes peuvent enfin circuler dans l'enceinte du Palais Bourbon pour accéder aux informations au plus près de la source. Une nouvelle forme de relation entre députés et journalistes se met alors en place durant ces premières années de la III ${ }^{e}$ République, à la méfiance et au conflit succèdent des relations personnelles, d'échanges de services et de coopération. Les activités du Parlement y gagnent une visibilité accrue et la presse acquiert une complète légitimité à présenter au public les affaires parlementaires. Néanmoins, pour Marc Martin (1997, p. 149), ce régime, en cantonnant les journalistes à l'écart de nombreux lieux et moments de la vie parlementaire, les place « dans une position d'infériorité, sinon de subordination du moins de dépendance $»^{5}$. C'est à l'issue de la Seconde Guerre mondiale que s'ouvre la troisième période, tandis que la télévision et la radio viennent bousculer l'équilibre et les routines en place. Jusqu'alors les débats en séance publique n'étaient retransmis que de manière différée sous forme de discours transcrits. Le son et l'image étendent dorénavant le champ de ce qui est perceptible par le public et offrent aux députés les moyens de renouveler leurs stratégies de mise en scène. Les débats parlementaires sont diffusés à la radio dès 1946 et la première autorisation à les filmer date de 1956. En 1981, les séances de questions au gouvernement sont retransmises à la télévision, en direct (Garrigues, 2007). En 1999, une chaîne de télévision spécifique, LCP, est créée pour rendre compte au plus grand nombre, en direct et de manière continue, des activités parlementaires (Hourquebie, 2013). Dès le début, l'Assemblée nationale s'efforce de canaliser ces nouveaux supports d'information et déploie des dispositifs de contrôle efficaces pour conserver la maîtrise de l'information produite en son sein. Cependant, la faible audience des retransmissions des débats, dans un régime devenu semi-présidentiel, amène progressivement la

5. Cité par Le Torrec (2005, p. 194). 
presse à recentrer sa programmation sur des émissions politiques réunissant notamment des journalistes et des députés en face à face. Aujourd'hui, les activités parlementaires ne constituent plus le cœur de l'actualité politique couverte par les médias grand public. Le rapport de force entre les députés et les journalistes se renverse alors, l'effort d'exposer l'activité parlementaire au public incombe désormais à l'institution, et plus particulièrement aux députés. C'est ce qui fait dire à Le Torrec (2005, p. 205) que « la médiatisation des activités parlementaires ne dépend désormais plus tant de l'accès donné aux journalistes que de la valeur informationnelle dont elles sont dotées ».

C'est dans cette perspective historique que se place l'analyse qui va suivre. Le développement des médias audiovisuels ces dernières décennies est venu modifier les modes d'exercice du pouvoir législatif. D'une part en amenant l'institution parlementaire à reconfigurer les frontières de son propre régime de visibilité publique et, d'autre part, en influant sur la manière dont les représentants politiques apparaissent au reste de la société. Ce phénomène, qui peut être qualifié de «nouvelle visibilité » médiatisée à la suite de John B. Thompson (2005), a pris encore une nouvelle dimension avec le développement d'Internet et des médias numériques. De son côté, l'institution qu'est l'Assemblée nationale française y a vu son intérêt, en faisant des médias sociaux tels que Twitter et Facebook un canal supplémentaire pour promouvoir l'activité parlementaire dans l'espace public (Leston-Bandeira et Bender, 2013). Les députés français de leur côté n’ont pas non plus attendu pour expérimenter ce que ces nouveaux médias pouvaient leur apporter en termes d'exposition médiatique. Il faut dire que, pour ces derniers, l'exposition médiatique n'est pas nécessairement acquise. Ils ne sont, sauf exception, pas de ces hommes politiques qui se voient assurés d'un accès quasi routinier à la visibilité publique. Seule une élite d'entre eux bénéficie d'une couverture médiatique permanente et toujours acquise, parce qu'ancrée dans des routines institutionnelles et médiatiques. Les députés sont, à leur niveau, eux-mêmes engagés dans cette « lutte pour la visibilité » que décrit Olivier Voirol (2005), c'est-à-dire doivent recourir à des procédés de visibilisation qui dérangent les arrangements en vigueur. Le constat que faisait Jean Charron (1994) à 1'Assemblée nationale du Québec à la fin des années 1980 se vérifie ici aussi : au Parlement, «c'est souvent la source qui quête de la publicité et non le journaliste qui quête de l'information ». Remarquons que la publicité parlementaire se joue ainsi aujourd'hui sur deux plans. Elle concerne premièrement l'effort de l'institution pour médiatiser, par ses propres canaux d'information, les activités délibératives et législatives qu'elle accueille et ainsi leur 
assurer une visibilité publique. Elle désigne dans un second temps la stratégie de communication déployée par les députés qui cherchent à convaincre et à susciter l'adhésion, c'est-à-dire à faire ressortir une image positive de leurs positionnements dans le débat public (Urvoas et Alexandre, 2012, pp. 207208), et qui pour ce faire sont en quête des fenêtres d'exposition médiatique que peuvent leur fournir des journalistes. Or, contrairement à la publicité parlementaire institutionnelle, la visibilité publique des députés se joue dans un espace médiatique fortement concurrentiel.

Ainsi, l'usage de Twitter sera ici envisagé dans un contexte historique et politique particulier, comme une réponse de députés en quête de visibilité à des logiques médiatiques et institutionnelles jugées contraignantes. La prééminence, dans la couverture médiatique de l'actualité parlementaire, que se réservent les quelques députés occupant une position forte dans l'appareil politique ou dans l'institution, est un exemple de ces contraintes. La préférence des journalistes pour l'événement politique plutôt que pour les travaux délibératifs et législatifs ordinaires en est un autre exemple. La finalité visée est pour eux de rééquilibrer un rapport de force qu'ils considèrent comme insuffisamment avantageux. Le journaliste serait-il ainsi en position privilégiée vis-à-vis du député ? Les faits ne semblent pas l'indiquer, mais donnent plutôt à voir le maintien d'un certain équilibre dans la mesure où " chacun essaie d'exercer une influence sur le comportement de l'autre et, finalement, tente d'acquérir et de maintenir, à l'encontre de l'autre, une maîtrise sur le mécanisme de construction de l'actualité politique » (Charron, 1994, p. 11). Leur relation mutuelle doit plutôt être comprise comme l'expression d'une tension permanente. De manière contradictoire, la production de l'actualité politique suppose à la fois qu'ils collaborent étroitement et qu'ils jouent leur rôle en maintenant une distance. Ce rapport basé autant sur la coopération que sur le conflit a amené Jean Charron à concevoir les échanges entre députés et journalistes à l'aune du principe de négociation, les fenêtres d'exposition médiatique se marchandant contre des informations évaluées selon leur valeur dans l'espace médiatique. Dans cette idée, la notion de séduction, ensuite, sera ici utile pour éclairer la manière dont les sphères privées et publiques des députés et des journalistes s'entremêlent dans des stratégies individuelles, où chacun tente à son niveau de résoudre cette tension. Ainsi, ce texte a pour première finalité la description de ces stratégies menées au quotidien par les députés envers les journalistes. En second lieu, ce texte a pour objet de montrer en quoi les nouveaux usages médiatiques, portés par Twitter notamment, ne viennent pas remettre en question ce jeu d'influence, mais le renouvellent et le recomposent dans de nouveaux espaces. La matière première de ce travail 
s'appuie sur une enquête ethnographique à l'Assemblée, menée notamment en tant que collaborateur parlementaire. Huit témoignages de députés, journalistes et collaborateurs ont été sélectionnés ici, conduits principalement durant les six premiers mois de l'année 2014.

\section{LES NÉGOCIATIONS EN SALLE DES QUATRE COLONNES}

La salle des Quatre Colonnes est le lieu traditionnel des rencontres entre les députés et la presse dans la vie politique française. Attenante à l'hémicycle, elle reste aujourd'hui le cadre privilégié de leurs interactions dans l'enceinte $\mathrm{du}$ Palais Bourbon. Sous les yeux des huissiers veillant au respect des quelques règles encadrant les échanges, les journalistes peuvent dans ce lieu à l'exiguïté notoire poser leurs questions aux députés ou recueillir leurs confidences. Ces échanges peuvent en théorie se faire tous les jours de la semaine en période de session parlementaire, pourtant c'est principalement les mardis et mercredis que cette salle remplit sa fonction, lors des deux séances hebdomadaires consacrées aux questions au gouvernement. À leur sortie de l'hémicycle, qu'ils désirent ou non rencontrer la presse, députés et ministres traversent cette salle ${ }^{6}$. Ce qui donne lieu pour l'essentiel à des interviews télévisées ou radiophoniques, mais également à des échanges informels non enregistrés. Au centre de la salle, des canapés de velours rouge sont mis à disposition de ceux qui désirent prendre le temps de discuter confortablement, signe d'un espace pensé pour des rencontres, en tant que relations de coopération fructueuses et durables, plutôt que pour des interactions fugaces et impersonnelles entre des individus sans accointance. Cette salle s'anime au gré des séances, si bien qu'elle peut être considérée comme une caisse de résonance de ce qui se passe dans l'hémicycle (Huet, 2011).

Jean-Jacques Urvoas et Magali Alexandre, respectivement député et collaboratrice de député au moment de la parution de leur ouvrage Manuel de survie à l'Assemblée nationale (2012), expliquent qu'un incident de séance suffit à voir la presse se précipiter sur quelques têtes connues dans l'espoir de recueillir la petite phrase qui fera sensation le reste de la journée. Ce qui les amène à regretter que les journalistes restent trop peu dans les tribunes qui leur sont réservées au-dessus de l'hémicycle, pour préférer la salle des Quatre Colonnes

6. Une issue alternative existe toutefois pour celles et ceux qui voudraient éviter de rencontrer la presse à un moment inopportun. 
(Urvoas et Alexandre, 2012, pp. 222-223). Effectivement, ce lieu est souvent celui de la concision, de la rapidité et de l'instantanéité pour des députés qui s'adressent à des journalistes avides d'incidents, d'accidents ou de péripéties. Pourtant, on voit aussi régulièrement des députés s'y rendre, un amendement à la main, pour une discussion technique avec un journaliste spécialisé, sans attendre une exposition médiatique immédiate. Les échanges entre députés et journalistes qui s'y tiennent ne débouchent pas tous sur une interview, et si la salle des Quatre Colonnes en est le lieu privilégié, c'est qu'elle constitue d'abord un espace d'interaction unique.

L'analogie avec une halle de marché, que nous propose Hélène Bekmezian (2013), journaliste parlementaire, met bien en lumière ce point. Partant du constat que la salle des Quatre Colonnes est le cadre d'un ballet bien réglé qui accueille des « échanges en tout genre [...] avec de bons clients, de bons commerciaux, des détaillants, et même quelques illégaux... », elle s'attache à en décrire une typologie des rôles. On y voit des journalistes-clients, qui achètent des informations, des commentaires, des analyses auprès de députés-vendeurs, avec des fenêtres d'exposition médiatique comme monnaie d'échange. Mais, dans cette halle de marché, tous ne sont pas égaux. De bons vendeurs fournissent de la marchandise qui n'est jamais décevante, calibrée pour les formats télé et radio, et ne manquent jamais d'acquéreurs. D'autres sont de tels commerciaux qu'ils sont en mesure de toujours trouver un micro pour faire passer un message, et sont disposés à répondre à peu près à n'importe quelle question. Sinon, en luttant sans relâche pour attirer le chaland, certains parmi les plus doués des seconds couteaux parviennent à jouir d'une audience médiatique inversement proportionnelle à leur poids politique. Enfin figurent les quelques privilégiés qui se trouvent sous les feux de l'actualité et qui s'attachent à prolonger l'état de grâce. Traverser la salle régulièrement, sans s'arrêter malgré les sollicitations, est une bonne technique pour entretenir une convoitise. Ce ballet est cependant réservé à une minorité. Le plus grand nombre des députés ne s'y sent pas à l'aise, tant et si bien qu'on les y voit peu. Il y a ceux qui refusent tout net de participer à ce qui est perçu comme une mascarade. Pour d'autres, cette mise à l'écart ne relève pas d'un choix, ils évitent les lieux en regrettant leur manque de répartie face aux caméras, ou s'y attardent au contraire en espérant qu'on les sollicite. En somme, il faut bien voir que ce jeu médiatique n'est réservé qu'à une élite : « les ministres, les élus vedettes, les grandes gueules » (Zineb, 2012). Pour les autres, seules deux stratégies sont possibles pour percer : demeurer dans les parages dans l'espoir d'une heureuse occasion ou jouer la provocation. Combien de députés tiennent alors un étal dans cette halle de marché ? Ce 
nombre est difficile à quantifier précisément. Une petite centaine d'individus serait néanmoins un ordre de grandeur plausible, obtenu par recoupement de plusieurs témoignages. Les presque cinq cents autres, c'est-à-dire huit députés sur dix, constituent le « ventre mou » de l'Assemblée, selon l'expression consacrée.

Cette description en termes de relations marchandes évoque un jeu qui oppose et unit à la fois journalistes et députés. D'un côté, la masse de journalistes présents en salle des Quatre Colonnes constitue une indiscutable opportunité médiatique pour députés en mal d'influence, tant et si bien qu'aujourd'hui, d'après Danièle Breem, doyenne des journalistes parlementaires, certains « se mettraient à poil pour passer au 20 heures $»^{7}$. D'un autre côté, les députés français devinent bien que les journalistes sont pris eux-mêmes dans une course à l'audience, si bien que beaucoup d'entre eux ne prennent pas le risque de ne rien avoir à transmettre à leur rédaction (Urvoas et Alexandre, 2012, p. 224). Tous tentent dans ce jeu de tirer leur propre épingle, en s'efforçant de s'associer au partenaire qui leur offrira le maximum de profit. Ce jeu relève d'une lutte qui, selon Charron, s'appuie avant tout sur des négociations. Ces négociations doivent être entendues à la manière de Christophe Dupont (1982), comme l'" activité mettant face à face deux ou plusieurs acteurs, qui, confrontés à des divergences et se sentant interdépendants, choisissent la recherche effective d'un arrangement pour mettre fin à cette divergence et ainsi créer (ne fût-ce que temporairement), maintenir ou développer une relation entre eux $»^{8}$.

Ces stratégies interindividuelles consistant à s'éviter, se faire désirer, se faire confiance, marquent une tension permanente qui, si elle ne cesse de se réactualiser au gré des lieux, des moments et des rencontres, est particulièrement saillante en salle des Quatre Colonnes. Les détours conduisant à ce qu'une relation privilégiée, ponctuelle et circonstanciée s'établisse entre deux individus relèvent en grande part de non-dits. Un second journaliste propose une autre analogie très explicite sur ce plan :

C'est vraiment [...] un lieu d'échange d'information et un lieu de contact [...] l'image que j'ai depuis assez longtemps, depuis que j'y suis, c'est l'image du

7. Propos cités par Haguet (2012). Voir aussi sur ce point la course à l'image des députés décrite par Abélès (2001, p. 293).

8. Cité par Jean Charron (1994, p. 26). 
cruising . [...] En fait, t'es dans un lieu de drague. [...] Voilà, t'as une espèce de circulation des corps, assez épuisante, fatigante, mais qui t'apprend des choses, tu dois apprendre à refuser de façon polie un peu, c'est exactement comme dans les jeux de séduction. [...] Des gens te saluent de loin, des gens t'approchent, des gens t'alpaguent, des gens sont relous, des gens veulent pas te lâcher, t'as des gens pour qui c'est ta priorité de les voir, mais c'est un peu comme... le mec que t'as repéré depuis le début et que tu sais que tu finiras avec lui ${ }^{10}$.

\section{LA SÉDUCTION COMME MODALITÉ D’INTERACTION}

Ce dernier témoignage, où l'on voit se surajouter une dimension corporelle au concept de négociation, fait écho aux travaux de Cyril Lemieux (2000). La question de la collusion entre les journalistes et la classe politique le conduit aux mêmes observations, il y a de toute évidence dans les relations entre les députés et les journalistes, une place pour le corps, pour l'affect, pour l'humain. En salle des Quatre Colonnes, « on s'embrasse, on se tapote l'épaule, on se tutoie, on rit ensemble, on s'offre des cigares, on s'invite à déjeuner » (Lemieux, 2000, pp. 127-128). Pour l'expliquer, il distingue les situations publiques des situations privées, dans lesquelles les règles de conduite en vigueur (la « grammaire ») diffèrent. Il détaille ainsi des règles de conduite propres aux situations qui ne sont pas publiques (comme en salle des Quatre Colonnes), et dans lesquelles il ne s'agit pas de préserver une distance, mais au contraire de s'engager, de manière immédiate, totale et non médiatisée (la « grammaire naturelle »). Les motifs d'engagement dans de telles relations privilégiées sont nommées par Lemieux (2000, p. 153) des «attractions », aux sens où les députés et les journalistes doivent « par-delà ces tâches qu'ils ont à accomplir et ces rôles qu'ils ont à assumer, [se montrer] mutuellement profonds, attirants, d'agréable compagnie ou doués de charme ».

C'est en ce sens précisément que s'envisage ici le concept de séduction, conjointement à celui de négociation. Cette séduction n'est pas amoureuse, elle n'implique aucune emprise d'une personne sur une autre, elle n'est pas un rapport de domination. La séduction n'est pas non plus celle que l'homme

9. Le cruising, dans l'imagerie de la culture gay, définit l'activité de recherche d'un partenaire sexuel - usuellement anonyme, inconnu et pour une occasion unique - dans un lieu spécifiquement public.

10. Entretien du 13 février 2014. 
politique cherche à exercer sur l'opinion au travers des médias, bien qu'elle n'y soit pas tout à fait sans lien. La séduction n'est pas l'arme de celui qui cherche à abuser de la naïveté de ses auditeurs, elle n'est pas en elle-même « ce qui ôte au discours son sens et le détourne de la vérité ${ }^{11}$, comme peut l'écrire Jean Baudrillard (1979). La séduction est ici vue comme une forme de négociation parmi d'autres, préalable à une rencontre, quelle que soit la finalité de celle-ci (amoureuse, sexuelle, professionnelle...), qui a ceci de spécifique que son langage passe en partie par une proximité corporelle et une mise en scène de soi. Il faut souligner, avec Gilles Boëtsch et Dorothée Guilhem (2005, p. 180), que la notion de séduction reste encore en sciences humaines généralement employée dans un sens commun. Il y a pourtant « une confusion de sens [qui] provient du fait qu'elle se trouve associée à d'autres notions telles que la sexualité, le fantasme, l'érotisme, la drague ou le charme ». En effet, d'un point de vue conceptuel, rien ne justifie que la séduction soit irrémédiablement rattachée au domaine de l'intime. Au contraire, on ne peut faire l'économie d'interroger ses manifestations et ses usages dans la vie publique, collective, précisément pour le rapport au corps qu'elle implique. Ce témoignage qui nous propose de concevoir la salle des Quatre Colonnes comme un « lieu de drague " doit alors retenir notre attention. On nous invite à y voir un ensemble d'interactions, dans un espace à l'écart de toute visibilité publique, qui se tissent sur un mode de communication spécifique, constitué à la fois d'actes de parole, d'attitudes gestuelles et d'affects. On peut alors faire l'hypothèse que cet espace a pour fonction précisément d'encadrer, de régir, voire de favoriser ces interactions privilégiées entre députés et journalistes, au point qu'il y règne une « proximité physique qui confine parfois à la promiscuité $\gg{ }^{12}$.

Les cas de séduction des journalistes par les politiciens ne manquent pas dans l'histoire récente. Christian Delporte (2011) nous en donne un aperçu dans un ouvrage qui, dans sa globalité, nous montre de façon saisissante combien séduire l'opinion passe d'abord par la séduction des journalistes. Politiciens et journalistes ne cessent d'interagir sur le mode de la séduction, d'autant plus dans une période où les sphères médiatique et politique tendraient à fonctionner comme une seule entité (Gingras, 2009). Remarquons toutefois que tous les exemples évoqués vont dans le même sens, à savoir que ce sont les

11. Cité par Christian Delporte (2012, p. 11).

12. Échange par courriel avec un journaliste politique, ancien assistant parlementaire, le 7 juin 2014. 
politiciens qui usent d'abord de la séduction envers les journalistes. Il faut ici prendre ses distances avec un discours tenu par l'institution sur elle-même. En ce qui concerne la situation parlementaire, ce qui se joue en salle des Quatre Colonnes doit aussi être vu sur un plan symbolique. Les jeux de séduction qui entourent les échanges procèdent d'une actualisation continue de la doctrine républicaine, qui attend du représentant de la souveraineté populaire qu'il soit la figure centrale du système politique. Ainsi, il est dans l'intérêt de l'Assemblée nationale de présenter les députés comme des maîtres séducteurs, à l'initiative et dictant le rythme de la vie politique. En haut de l'estrade figure ainsi symboliquement le peuple souverain, qui impose ses règles au pouvoir médiatique et qui se positionne en concurrence directe avec le pouvoir exécutif, donc en position de pouvoir. Les journalistes ne seraient plus dans cette optique que des courroies de transmission d'une information politique recueillie passivement.

$\mathrm{Au}$ ras des faits, les choses sont moins simples. Certes, il est indéniable que les journalistes sont un des vecteurs de la publicité parlementaire institutionnelle, autant que des stratégies individuelles de communication politique, par le fait même de participer au ballet de la salle des Quatre Colonnes. C'est effectivement ainsi qu'ils entretiennent à leur niveau les conditions d'existence des députés, en créant les conditions de leur visibilité médiatisée. Ils se font autant le relais de leur mise en représentation que le relais du contenu de leur discours. Pourtant, les journalistes ne sont pas démunis pour ce qui est d'obtenir des confidences, des aveux, en usant eux-mêmes de séduction, c'est-à-dire en négociant les interactions par un langage du corps, du nonverbal. Pour en avoir un aperçu, il faut par contre quitter la salle des Quatre Colonnes, et s'intéresser aux stratégies mises en œuvre, dans des espaces plus neutres, qui sont moins au service exclusif de l'image positive des députés. D'autant que pour s'engager totalement dans une relation privilégiée, il faut savoir faire preuve d'intimité, c'est-à-dire se rencontrer " en aparté et à l'écart des autres », sans que les attributs du journaliste (calepin, micros) introduisent une distanciation inopportune (Lemieux, 2000, pp. 156-158). Un journaliste politique, ancien assistant parlementaire, m'explique de ce point de vue que les marchés secondaires, beaucoup plus discrets, ne doivent pas être négligés :

Comme les auditions et travaux de commissions sont ouverts à la presse, il est possible, avant et après, dans les couloirs, voire dans la salle de la commission, d'échanger avec les politiques, dans des conditions beaucoup plus paisibles. On est souvent seul avec le parlementaire, sans stress, sans foule. On a le temps de discuter. C'est surtout valable pour la presse écrite, où on 
note un échange verbal, sans avoir besoin de filmer ou de prendre un son. [...] Quand on sait exactement ce qu'on veut et qui on veut voir, cela peut être très rentable... Aux Quatre Colonnes, on a les journalistes politiques, en sortie de commission, on a la presse spécialisée (à qui il ne viendrait jamais à l'idée d'aller aux Quatre Colonnes, comme il ne viendrait pas à l'idée d'un journaliste politique, sauf sujet sensible, d'aller faire les sorties de commission) $)^{13}$.

Ou cette journaliste parlementaire qui m'expliquait :

J'installe un rapport agréable avec eux. [...] C'est plus facile pour ramener de l'information. Je vois au service politique [du journal], il y a des gens qui ne sont pas du tout dans ce rapport-là aux sources. Parce qu'ils disent : "Moi je suis hyper-indépendant, il n'est pas question qu'on blague ensemble !" Mais je vois que ça leur pose problème, surtout en politique, parce qu'il y a des [informations] qu'ils n'ont pas $^{14}$.

Entretenu dans le registre de la convivialité professionnelle, l'engagement dans la relation n'est pas gratuit, mais se fait dans l'optique de rendre plus fluide la circulation de l'information. La question éthique est ici centrale pour les journalistes. Ceux que j'ai rencontrés sont très soucieux de m'expliquer quels garde-fous leur permettent d'éviter le piège d'une coopération qui deviendrait collaboration, ou leur assurent une protection contre les tentatives dissimulées d'instrumentalisation. Ces réticences à s'engager totalement dans une relation privilégiée, ces efforts d'autocontrainte que les journalistes s'imposent à eux-mêmes pour endiguer une attraction jugée trop périlleuse, sont décrites par Lemieux (2000, pp. 170-171) comme des « répulsions ». L'articulation entre l'attraction et la répulsion dans la relation aux hommes politiques est une problématique permanente pour les journalistes. Une problématique qui, par ailleurs, ne semble pouvoir être résolue qu'au cas par cas, dans un effort continu qui laisse une large place à l'auto-évaluation, et donc d'une manière qui ne peut qu'être propre à chacun. Cette capacité d'articulation est ainsi la première compétence à acquérir pour un journaliste politique, c'est-à-dire être capable de mettre en place des stratégies de l'ordre de l'empathie, de la compassion, destinées à créer des relations privilégiées, tout en préservant une certaine distance. À l'Assemblée, cette capacité s'avère particulièrement nécessaire dans la mesure où :

13. Échange par courriel avec un journaliste politique, ancien assistant parlementaire, le 7 juin 2014.

14. Entretien du 21 mai 2014. 
C'est quand même un endroit où plein de mecs dorment sur place, l'humain rentre forcément en compte! Ils passent leurs nuits ici, moi j'en ai vu pendant le débat « mariage » venir avec leurs gosses parce qu'ils ne savent pas comment les faire garder... donc c'est impossible de faire une stricte séparation humain/politique. Je veux dire : j'ai pratiquement habité avec eux pendant qu'on a fait les débats sur le mariage ${ }^{15}$ !

Alors, le journaliste parlementaire, détaché sur place, dispose d'un avantage certain sur le journaliste politique dont la présence n'est qu'épisodique. Le premier est spécialisé, il connaît bien le terrain, les mécaniques institutionnelles, mais surtout il passe du temps sur place, il participe à la vie interne à l'institution, il vit le même rythme au quotidien. La coprésence reste le meilleur moyen pour faire venir à soi l'information ${ }^{16}$. Le journaliste parlementaire « est de la maison », selon l'expression consacrée, sa mission est de participer à l'ordinaire des activités de l'Assemblée pour faire le relais avec ses collègues journalistes politiques. On notera qu'un tel fonctionnement montre que l'entraide et l'entente entre journalistes employés par des maisons de presse différentes sont la norme. Les journalistes de l'AFP, de Reuters, du Monde et du Figaro sont particulièrement positionnés comme des référents et des dispensateurs de conseils pour les collègues qui ne s'intéressent au Parlement qu'épisodiquement, ce qui n'empêche pas qu'ils puissent se réserver la primeur de certaines informations ou de certains contacts.

S'il y a une relation de séduction entre les députés et les journalistes, c'est au sens où l'interaction tend à s'établir sur une forme de reconnaissance affective, qui joue ici sur l'effet grégaire, dans la visée de réduire la distance qui les sépare. L'information circule mieux quand elle circule dans un entre-soi. Toutefois, c'est un mode de fonctionnement qui implique, dans l'intérêt des deux parties, d'éviter les sujets qui fâchent. La séduction est une modalité d'interaction efficace dans ce contexte, mais qui a ses zones d'ombre :

Les infos que je ne fais pas [...] c'est les trucs vraiment contre l'institution, c'est-à-dire par exemple, quand je fais des papiers sur la réserve parlementaire. [...] C'est des trucs, comment dire, après ça peut me poser un vrai problème. [...] C'est pas [seulement] de taper sur tel politique [...] ça fait partie du jeu, tout le monde l'accepte. Mais quand j'attaque l'institution... Parce qu'ils ont beau être de droite, de gauche, ils sont tous très solidaires entre eux. [...]

15. Entretien du 21 mai 2014.

16. Ce que note aussi de son côté Cyril Lemieux (2000, p. 153). 
Et ça, je ne le fais pas, par facilité, parce que je vais m'en prendre plein la gueule, parce que j'ai pas le temps, par flemme aussi un peu, et c'est une des lacunes de mon boulot ${ }^{17}$.

\section{UN JOUR SURVINT TWITTER ET LE DÉPUTÉ DEVINT MÉDIA}

La salle des Quatre Colonnes est donc un grand marché où se négocient des informations et des fenêtres d'exposition médiatique. La séduction est une stratégie de négociation qui préside à l'échange de ces deux types de ressources, dans cette salle d'abord, mais aussi de manière plus générale à l'Assemblée. Comment Twitter, dont l'usage par les députés est incontournable depuis septembre 2012, la première rentrée parlementaire de la quatorzième législature, s'intègre-t-il alors, se positionne-t-il dans ces interactions ? Une anecdote d'un journaliste parlementaire de l'AFP illustre de manière symptomatique quels enjeux a cristallisés Twitter dans la relation entre journalistes et députés :

Moi j'ai quand même un souvenir cuisant avec Twitter, c'était en juillet 2012. Le groupe socialiste se réunissait pour élire son candidat à la présidence de l'Assemblée nationale. [...] Et il devait par ailleurs élire son président. [...] Je m'étais mis d'accord avec un ou deux députés, et puis avec l'attaché de presse, pour qu'ils m'envoient par SMS les résultats. Sauf que des députés se sont mis à tweeter. [...] Les premiers tweets sur l'élection de Le Roux [pour la présidence du groupe] sont donnés avant l'alerte de l'AFP. Forcément. Envoyés directement par un député... [alors que] l'alerte va être publiée quelques minutes après, le temps de recevoir le SMS, plutôt deux pour être sûr qu'il n'y a pas une erreur. Et, quelqu'un tweete : " Il y a vraiment plus besoin de l'AFP, puisqu'on a tout sur Twitter. » Ça m'a beaucoup agacé. [...] Puis, j'ai reçu [...] un SMS me disant que Bartolone était en tête [pour la candidature à la présidence de l'Assemblée]. J'étais tellement tendu, j'ai tellement eu envie de faire vite, que j'ai envoyé que Bartolone était élu. Quand je me suis rendu compte de mon erreur, j'ai réussi à arrêter à temps la dépêche sur un des fils de l'AFP avant qu'elle n'arrive au client. Elle est quand même, chez certains clients, arrivée par erreur. Heureusement, Bartolone finalement a été proclamé élu sans qu'il y ait besoin de deuxième tour. Et ça m'a servi de leçon ${ }^{18}$.

17. Entretien du 21 mai 2014.

18. Entretien du 13 février 2014. 
Ce témoignage montre que l'équipe AFP de l'Assemblée nationale, principal acteur de presse parlementaire française avec ses quatre journalistes à plein temps détachés sur place, s'est vue avec Twitter mise en concurrence avec d'autres relais d'information. Jusqu'ici leurs usages, pour contourner les restrictions d'accès qui sont imposées aux médias dans les couloirs et les salles du Palais, consistaient à négocier des informations en amont, auprès d'informateurs privilégiés, c'est-à-dire avant même leur mise en circulation : ici le résultat à venir d'une élection, mais aussi l'annonce d'un accord en commission à huis clos, l'ordre du jour de la réunion d'un groupe politique, ou l'audition privée d'une personnalité publique. Les SMS circulaient ainsi entre, d'un côté, ceux qui se faisaient les oreilles et les yeux de la presse parlementaire, à savoir des députés, des assistants parlementaires, des administrateurs du service de presse de l'institution et, de l'autre côté, les journalistes qui se faisaient éventuellement le relais ensuite auprès de confrères moins intégrés dans la maison. Twitter vient bousculer ces dispositifs de collecte, en permettant à celui qui détient une information d'un quelconque intérêt, de prendre de vitesse l'AFP par une publication qui ne mobilise aucun intermédiaire. Pourtant, le tweet et l'alerte AFP sont des supports de publication au format et à la fonction comparables. Quand le tweet est pensé comme une réponse à la question What's happening? ${ }^{19}$, l'alerte permet de couvrir un événement en cours, dans l'attente de la rédaction d'une dépêche au contenu plus fourni. Les deux supports répondent au besoin d'une information instantanée et condensée. Alors, l'argument de la vitesse de publication sollicité par ce journaliste pour expliquer que les députés puissent se détourner de l'AFP a une portée limitée. Le message à l'origine de son énervement peut être lu d'une seconde manière. S' « il [n']y a vraiment plus besoin de l'AFP, puisqu'on a tout sur Twitter », peut-être est-ce aussi que ce support permet de s'affranchir d'intermédiaires jugés envahissants ${ }^{20}$. En ce cas, le tweet surpasserait l'alerte AFP en ce qu'il est publiable par tout un chacun, et donc offre une marge de liberté.

Pour reprendre l'analogie du grand marché de l'Assemblée, avec Twitter, la circulation de l'information dans l'espace public s'est libéralisée. L'AFP a perdu sa position privilégiée, de monopole pourrait-on dire, ou plus exactement de premier relais. L'AFP s'était fait une spécialité de suivre en continu

19. Slogan commercial de Twitter depuis 2009, remarquable en ce qu'il reprend une question clé sur le journalisme professionnel, comme le notent Nicolas Pélissier et Gabriel Gallezot (2013, p. 27).

20. Nicolas Pélissier et Mamadou Diouma Diallo évoquent à ce titre un phénomène de dispersion du journalisme (2013, p. 173). 
le processus d'élaboration de la loi, en couvrant toutes les séances publiques, et surveillait les travaux des commissions. Elle faisait son commerce sur la dimension la plus méconnue, la plus austère et la plus technique de la vie parlementaire, et laissait aux confrères le soin de mieux couvrir la salle des Quatre Colonnes ainsi que les débats spécialisés et marquants de l'actualité politique. Ceci lui conférait une réputation de rigueur et de crédibilité, dont les députés savent d'ailleurs encore user selon leur stratégie du moment, en fonction du type de message à faire passer. Subitement, en mi-2012, au moment précis du changement de législature, Twitter vient bousculer cet équilibre. À cette date, la moitié des députés nouvellement élus disposent de leur compte personnel sur la plate-forme (Chibois, 2014) et en juillet 2014, deux ans plus tard, ils sont soixante-quinze pour cent. Tous ne publient pas de tweets, beaucoup utilisent leur compte d'une manière passive ${ }^{21}$, c'est-à-dire pour simplement manifester une présence, pour pouvoir ainsi être interpellé par un potentiel interlocuteur et pour suivre ce qui se dit sur le mot dièse \#directAN. Toute personne peut en effet aujourd'hui, sans intermédiaire autre que la plate-forme technique, avoir une idée assez précise de ce qui se passe, ce qui se dit et ce qui s'annonce au cœur de l'actualité politique, pour peu qu'il s'attarde sur ce mot dièse.

Quelques mois plus tard, à l'occasion du projet de loi pour l'ouverture du mariage aux couples de même sexe, la France vit une importante controverse et cette plate-forme devient sur cette période le lieu privilégié où partisans et opposants expriment leurs convictions et organisent la mobilisation (Cervulle et Pailler, 2014). Twitter devient un espace médiatique central dans la vie politique française, et l'AFP ne peut qu'en prendre acte.

L'enjeu ici est celui de la primeur de l'information, le scoop ${ }^{22}$. Être à l'origine d'une information, c'est se positionner en tant que source incontournable de l'actualité politique, auprès du public, mais surtout auprès des confrères. Cela revient à s'assurer une audience dans un contexte de grande concurrence médiatique, ce qui se traduit à l'AFP par une clientèle. Ainsi, l'équipe AFP à l'Assemblée a vu, un temps, les conditions de son existence remises en question. À quoi bon maintenir une telle équipe AFP à l'Assemblée si elle n'est plus en mesure de proposer de l'information originale ? Faut-il laisser aux députés le soin de rendre compte de la vie parlementaire, sans qu'aucun

21. Au sujet de la variété des usages de Twitter par les députés, voir l'exemple du RoyaumeUni traité par Nigel Jackson et Darren Lilleker (2011).

22. Entretien du 13 février 2014. 
observateur extérieur ne demeure pour trier, contextualiser et hiérarchiser en continu l'information? En conséquence, actant qu'ils ne pouvaient rivaliser sur le terrain de l'instantanéité de l'information ${ }^{23}$, ils ont choisi de «lever le pied » et de se recentrer sur un rôle d'accompagnement de l'information.

Nous, notre force, ce qui nous permettra de défendre notre mission d'intérêt général, mais aussi notre raison d'être, c'est de pouvoir rapidement, moins rapidement que Twitter, c'est clair, mais quand même très vite, apporter quelque chose qui est fiable. [...] C'est important pour nous de garder la tête froide et d'accepter d'être en retard sur Twitter, parce qu'on apporte la fiabilité, des précisions ${ }^{24}$.

L'adoption de Twitter par les députés peut dès lors être analysée comme une stratégie de conquête de l'espace médiatique, à tout le moins pour ceux qui en sont d'ordinaire exclus. Les journalistes parlementaires rencontrés s'accordent sur le fait qu'ils ont " quand même un pouvoir énorme ", qui les place dans une position privilégiée à l'Assemblée. En tant que passeurs de l'information, ils jouent un rôle de filtre, mais l'indépendance professionnelle qu'ils s'échinent à préserver au quotidien ne satisfait pas toujours les intérêts politiques de tel ou tel député, ou de tel ou tel groupe politique. En tant qu'observateurs privilégiés de la vie parlementaire, ils créent littéralement la plus grande part de l'actualité politique, dans un processus performatif où une information ne devient un fait tangible qu'à partir du moment où elle est écrite par l'un des journaux institutionnels. Face à ce pouvoir, un député ne peut pas lutter à son échelle, il est contraint d'accepter les règles du jeu. Il doit savoir ménager les journalistes qui, eux seuls, sont en mesure de lui fournir l'exposition médiatique nécessaire à son influence. C'est ce que montre très explicitement cette anecdote, rapportée par une journaliste :

- Un jour, il y a [un député] qui n'arrêtait pas, qui est venu me voir une énième fois. Il est venu me dire : «Ah, Le Monde, vous allez encore dire du mal de moi... » J'ai dit : «Ah, c'est pas la peine, je m'en vais ! Et là, je me suis barrée, et il s'est mis à me courir après, en disant : « Non non non, c'est pas ce que j'ai voulu dire ! Allez-y posez votre question ! » Et là, j'ai compris le principe du rapport de force.

23. Pour être complet sur la thématique de l'information instantanée, il aurait fallu aussi évoquer l'essor récent des chaînes télévisées d'information continue. À leur niveau, elles ont participé à la mise en concurrence de l'information parlementaire publique et, donc, à faire que l'AFP à l'Assemblée se recentre sur ses activités.

24. Entretien du 13 février 2014. 
- Et pourquoi il a couru alors?

- Parce qu'il a toujours plus besoin de moi que moi j'ai besoin de lui. Le député a toujours plus besoin du Monde que Le Monde a besoin du député25.

L'atout principal d'un journaliste politique qui s'intéresse à l'Assemblée tient au nombre de sources à sa disposition, cinq cent soixante-dix-sept en théorie. La réalité est ici la même que celle que Jean Charron notait au Québec. La concurrence est rude pour les députés en ce qui concerne l'accès à l'espace du débat public, refuser un jour une fenêtre d'exposition médiatique revient à l'offrir à un collègue. À l'inverse, les journalistes à Matignon ou à l'Élysée, qui doivent travailler avec une source unique, ont beaucoup plus de difficulté à imposer leur indépendance. Dans ces hauts lieux du pouvoir exécutif, c'est la concurrence entre journalistes face à une source unique qui dicte le rapport de force. À l'Assemblée, le choix des sources laisse une marge de liberté confortable aux journalistes pour produire de l'information indépendante. On comprend alors les enjeux pour un député en quête d'une meilleure reconnaissance qui, avec Twitter, tente de prendre une distance avec ce mode de fonctionnement. Twitter paraît offrir aux députés une certaine position d'indépendance vis-à-vis des journalistes, une possibilité de contourner un rapport de force qu'il juge en sa défaveur, et de fait une forme d'autorité (Merzeau, 2013, p. 45). En cherchant à court-circuiter les logiques en place, le député cherche à se doter d'une plus grande initiative, et donc d'une plus large liberté de manœuvre, dans la publicisation de ses activités et de ses positionnements. Pour ce faire, il se fait lui-même média.

\section{TWITTER, UN APPEAU À JOURNALISTES}

Ce que disent les députés de leurs motivations à utiliser Twitter est une illustration éloquente de cette idée du député-média. Comme j'ai déjà eu l'occasion de le montrer (Chibois, 2014), l'adoption de cet outil par les équipes parlementaires a été dictée par une logique dite de l'appeau à journaliste, en référence à cet instrument utilisé pour attirer les oiseaux en imitant leur cri. En 2010, au temps des premières expériences, un assistant parlementaire me confiait quel en était l'état d'esprit :

[Pour nous, ] Twitter, c'est un usage très spécifique. C'est un appeau à journaliste.

25. Entretien du 21 mai 2014. 
- Qui marche bien?

- Du feu de dieu ! [...] [D'abord,] on leur a mis quelques bons petits trucs pour qu'ils suivent [ce député]. Et puis, ensuite à un moment, on a balancé la date et l'heure de la sortie de la décision du conseil constitutionnel sur [un texte] parce qu'on l'avait. [...] Et là, le nombre de followers... [il mime une progression fulgurante]. Heure par heure, ça montait par le biais des retweets. [...] Ensuite, on a commencé à live-tweeter les réunions : « en direct de la réunion de groupe, truc vient de se prendre un gros savon par [le Premier ministre] ». Et comme c'est 140 caractères, c'est suffisant pour appâter le journaliste, mais pas assez pour en dire de trop. Il sait, il sent qu'il y a un truc, mais il n'y a pas assez pour faire un article. Le résultat : [...] quand [ce député] live-tweete, dans l'heure qui suit, j'ai cinq coups de fil : « Bonjour c'est RMC ou I-Télé, est-ce que Monsieur le Député est en salle des Quatre Colonnes, on voudrait bien faire une interview de lui ?» [...] C'est de la notoriété. Le fait de passer dans les médias fait qu'il est connu, et donc plus il est connu, plus il est visible dans les médias, plus il a d'importance dans le microcosme politique ${ }^{26}$.

Également un député, en 2014 :

- Aujourd'hui, je ne fais plus de communiqués AFP. Je fais un tweet qui renvoie à mon blog.

- Et ça suffit?

- Hier j'en ai fait un [...], et j'ai renvoyé à mon blog où je disais du bien [d'un service de l'État]... quinze minutes après j'avais un mail d'Europel et de RTL me demandant si je pouvais le dire au micro ${ }^{27}$.

La formulation du principe de l'appeau à journaliste fait apparaître deux éléments importants. Premièrement, elle montre que Twitter s'intègre opportunément dans des usages médiatiques qui lui préexistaient. C'est un dispositif sociotechnique dont l'appropriation n'est pas la cause d'une évolution des usages, mais constitue plutôt une conséquence de ces usages qu'elle vient prolonger à sa manière. Deuxièmement, soulignons que la tentative des députés pour se doter d'une plus grande capacité d'initiative dans leurs relations avec les journalistes s'appuie sur des stratégies individuelles de mise en scène. Le fait que ce qui n'était initialement que des expériences médiatiques isolées ait rapidement et massivement été imité dans le contexte des élections

26. Entretien du 12 avril 2010.

27. Entretien du 22 avril 2014. 
législatives de 2012 ne doit pas être attribué au hasard. Les promesses de Twitter en termes d'exposition médiatique étaient telles que beaucoup ont trouvé une opportunité à saisir dans le cadre d'une stratégie communication de campagne.

À ce jour, il existe de multiples manières d'user de cet appeau, dont beaucoup sont vraisemblablement encore à inventer. Une typologie approfondie n'aurait pas sa place ici, toutefois en guise d'aperçu quelques pistes peuvent être évoquées. La première, historiquement parlant, semble bien être celle proposée dans cet avant-dernier témoignage, que l'on pourrait qualifier d'appeau-dépêche. Le député diffuse une information sur un événement, qu'il sait non couvert par d'autres, ce qui lui permet de se positionner en tant que source d'information incontournable. Ici, clairement, le député se met en concurrence avec l'AFP, en se faisant souvent vecteur de la publicité parlementaire par la diffusion d'informations qui ne concernent pas nécessairement son action politique propre. À ma connaissance, c'est une pratique médiatique inédite au Parlement, qui a été initiée par les députés Lionel Tardy, Yannick Favennec, Charles-Ange Ginesy qui se sont illustrés par des live-tweets polémiques entre janvier et juillet 2010. Il s'agit de se construire sur le long terme une audience (par l'accumulation de followers) dont il pourra bénéficier par la suite pour diffuser un message partisan. Dans d'autres circonstances, lorsque le député a besoin de se construire une stature nationale, l'utilisation de Twitter comme appeau-vitrine lui permet de maximiser son exposition, par la construction patiente d'un espace de rencontre pour une audience le plus souvent déjà acquise à la cause. En campagne électorale, on y relaie des prises de position, on y annonce des déplacements et on y diffuse des professions de foi. Hors campagne, quand elle assure la promotion de son travail législatif quotidien et use de la messagerie privée de la plate-forme pour gérer nombre de sollicitations dont elle fait l'objet, la députée Isabelle Attard en fait par exemple un usage de cet ordre. Ici, à bien des égards, le compte Twitter du député peut être assimilé à la permanence parlementaire en circonscription (Kerrouche, 2009), de par les fonctions qu'il remplit. D'une autre façon, un député ayant pour objectif d'occuper l'espace médiatique, non pas pour initier un débat de fond, mais pour publier des prises de position provocatrices, Twitter peut constituer un appeau-buzz. La plate-forme n'est ici qu'un support parmi d'autres qui vient se surajouter à l'existant, même s'il est peut-être plus aisé d'y voir une polémique prendre de l'ampleur. Ceci est d'autant plus vrai qu'aujourd'hui, les chaînes de télévision n'hésitent plus à filmer directement certains échanges de tweets. Les exemples sont ici nombreux, et certains comme la députée Nadine Morano, candidate malheureuse à sa réélection en 
2012, s'en sont fait une spécialité. Enfin, pour les députés impliqués dans le débat public, l'utilisation de Twitter comme appeau-porte-voix permet de faire la promotion de leurs billets de blogs par la publication d'accroches vendeuses. Il s'agit ici de susciter la curiosité et inciter à poursuivre la lecture sur un autre support plus propice à développer une argumentation. Les députés Xavier Breton et Jean-Jacques Urvoas figurent notamment parmi ceux-ci.

On pourrait croire qu'en se faisant média, le député se substituerait au journaliste. Au contraire, son usage de Twitter montre des pratiques variées qui ont pour point commun une stratégie visant à déclencher, à un moment ou à un autre, une exposition médiatique, ou a minima un dialogue avec un journaliste $^{28}$. Par exemple une interview dans la presse nationale ou régionale, une invitation à une émission télévisée ou radiophonique. Il faut dire qu'en dépit de leur caractère public, les échanges sur Twitter sont préservés de visibilité s'ils ne font pas l'objet d'une médiatisation. Pour quiconque se veut spectateur des échanges, le flot continu des tweets rend difficilement lisibles les discussions en les maintenant dans une zone de visibilité faible. Accessibilité de l'information ne rime pas avec visibilité de l'information. La twittosphère parlementaire est un " petit monde », théoriquement accessible à qui s'y intéresse, mais où, dans les faits, l'on demeure entre soi. Un petit nombre de députés et de journalistes politiques, pour l'essentiel, ne suivent l'activité que d'un petit nombre d'autres députés et journalistes, ensemble qui fonctionne en vase clos en formant un réseau d'affinités. C'est un entre-soi dans lequel sont négociées, de manière plus ou moins formelle, des fenêtres d'exposition médiatique à large audience contre des informations à forte valeur médiatique, objectif autour duquel les députés et les journalistes se retrouvent. C'est ainsi que, comme au Royaume-Uni (Jackson et Lilleker, 2011, pp. 100-101), cet entre-soi peut constituer une antichambre à la production de l'actualité politique : « Twitter has become a virtual 'smoking room' where they tease one another, gossip and occasionally score political points. » De la même façon qu'en salle des Quatre Colonnes, Twitter constitue un espace de sociabilité, où l'on négocie des contrats de médiatisation en même temps que l'on noue des rapports humains entre semblables. De la même façon aussi, cet espace de sociabilité s'appuie sur une coprésence, qui plus est hors de la visibilité

28. Sur ce point, au Parlement européen, Sandrine Roginsky et Valérie Jeanne-Perrier décrivent de manière similaire Twitter comme un dispositif par lequel les acteurs politiques ne s'adressent qu' « à des publics qui souhaitent être touchés, autrement dit principalement des citoyens engagés et des journalistes » (2014, pp. 103-104). 
publique, où l'on échange des flatteries " largely based on agreeing with other tweeters, and presumably building relationships with them ». Flatteries ( « ingratiation ») qui le plus souvent ne prennent pas la forme d'un échange conversationnel, mais se font plutôt sous la forme de mentions fréquentes, de retweets, de mise en favoris de certains tweets. De la même façon qu'en salle des Quatre Colonnes, les règles d'interaction entre députés et journalistes sur Twitter s'appuient sur le principe d'engagement personnel dans des relations privilégiées, que Lemieux nomme « grammaire naturelle».

Enfin, une fois de plus, comme en salle des Quatre Colonnes, on observe sur Twitter des efforts de mise en scène destinés à favoriser l'attraction mutuelle. Twitter constitue à ce titre un outil d' « impression management » (Jackson et Lilleker, 2011), c'est-à-dire qui permet au député de tenter d'influer sur la perception du public à son égard (en l'occurrence par l'intermédiaire des journalistes). Comme au Royaume-Uni, cette gestion de la « désirabilité sociale » constitue pour les députés français un outil de promotion de soi autant qu'un outil de mise en relation. Cette idée de l'appeau à journaliste nous ramène alors directement aux logiques de négociation et de séduction observées en salle des Quatre Colonnes. Twitter offre littéralement à ses utilisateurs, journalistes et députés, de « mettre en corps » (Casilli, 2010, p. 124) leur identité numérique et leurs sociabilités numériques, par la personnalisation de leur profil et la gestion fine des paramètres de leur propre exposition ${ }^{29}$. D'abord, en incitant à se décrire textuellement par des éléments autobiographiques et géographiques, ainsi qu'iconographiquement par une image censée figurer la personne titulaire du compte. Les députés usent largement de ces possibilités, et d'une manière plutôt homogène. Les images choisies sont constituées en très grande majorité d'une photographie réaliste de l'élu, en portrait ou en situation $^{30}$; les profils indiquent tous que leur possesseur est titulaire d'un mandat de député ainsi que la localisation de la circonscription qu'il représente. Un député me raconte :

[Twitter,] je ne m'en sers pas comme d'un outil d'échange, parce que je suis là aussi freiné par l'anonymat. [...] Moi, j'ai fait certifier mon compte de façon à

29. « Les médias électroniques se caractérisent par une grande variété d'indices symboliques, ce qui permet à ces nouveaux médias de reproduire certains traits typiques des interactions face à face » (Thompson, 2005, p. 198).

30. Comme j'ai pu le mettre en évidence concernant les photos utilisées par les députés pour illustrer leur profil (Chibois, 2013). 
ce que les gens sachent que c'est bien moi, c'est pas mes collaborateurs, c'est moi qui fais...

- C'est donc bien vous qui êtes derrière ce profil ?

- Ah, c'est moi qui tweete. Fautes d'orthographe comprises. Et tweet trop vite fait aussi une fois, et ça m'a vacciné. Donc maintenant j'annule plus de tweets que je n'en tweete ${ }^{31}$.

La certification du compte par Twitter participe de cette mise en corps. Il s'agit de garantir l'adéquation entre le profil et la personne, de garantir que - symboliquement au moins - la personne qui tweete est la personne qui prétend tweeter, c'est-à-dire l'authenticité d'une présence. Authenticité qui passe d'ailleurs aussi par des voies plus informelles, comme l'erreur, l'hésitation et les fautes d'orthographe. Ce profil est un avatar, au sens où le député y incarne sa fonction officielle par la mise en scène d'un personnage qui le représente à l'écran (Casilli, 2010, p. 124). Par l'entremise de cet avatar, il s'emploie à publier de l'information contextualisée. Ce sont les lieux et les personnes qu'il fréquente, les enjeux et les projets qui sont les siens qui vont déterminer le contenu de son information et, à ce titre, précisément, fera que cette dernière pourra constituer un intérêt pour les observateurs de l'Assemblée. Pour le dire avec les mots de Dominique Cardon (2010, p. 40), on voit ici comment Twitter participe d'un mouvement de " libération des subjectivités ». Les députés s'emploient par ce biais à se faire les reporters de la vie parlementaire, c'est-à-dire à donner à voir leur propre perception de l'Assemblée, en sachant que l'opinion publique et les journalistes politiques en sont aujourd'hui friands. Comme en salle des Quatre Colonnes, en somme, les députés se posent sur Twitter en maîtres séducteurs, au sens où ils s'emploient à faire venir à eux les journalistes pour ensuite négocier l'échange d'une information contre une fenêtre d'exposition médiatique.

\section{CONCLUSION}

Quelle place en définitive pour Twitter dans l'histoire des modalités de visibilité médiatisée des activités et des acteurs parlementaires? Du point de vue des journalistes, l'usage de Twitter a entériné, en même temps qu'il a contribué à le rendre possible, un mouvement en faveur d'une plus grande ouverture de l'espace médiatique. Pour ceux d'entre eux qui ne sont ni spécialisés

31. Entretien du 22 avril 2014. 
sur un thème ni détachés au Parlement, l'entretien d'un réseau de relations pour accéder à l'information n'est plus une nécessité fondamentale, puisque la coprésence nécessaire aux relations privilégiées n'est plus dépendante d'un ici et maintenant (Thompson, 2005). Du point de vue des députés, l'usage de Twitter apparaît comme une opportunité dans une lutte pour la visibilité, en offrant de contourner une logique médiatique sélective par laquelle seule une élite des députés bénéficie de l'essentiel de la couverture médiatique. Par la prise en main, en amateur (Cardon, 2010, p. 36), de la publicisation de leurs activités et positionnements, l'espace médiatique jusqu'ici matérialisé par la salle des Quatre Colonnes s'ouvre, en permettant une plus grande égalité d'accès de chacun à une visibilité publique médiatisée. L'espace de sociabilité que constitue Twitter offre aux députés une capacité renouvelée à initier des interactions avec les journalistes. Toutefois, si les positions ont été amenées à évoluer, les relations entre députés et journalistes, elles, n'évoluent pas à proprement parler, et il s'avère au final que peu d'éléments distinguent Twitter de la salle des Quatre Colonnes. En participant à désenclaver les relations entre la presse et l'Assemblée ainsi qu'à redéfinir certaines positions de pouvoir au sein de la vie parlementaire, on voit se prolonger une tendance initiée à la moitié du siècle dernier par laquelle la publicisation parlementaire s'éloigne encore un peu plus de l'hémicycle et des débats en séance. 


\section{RÉFÉRENCES}

ABÉLÈS, M. (2001), Un ethnologue à l'Assemblée, Paris, Odile Jacob.

BAUDRILlARD, J. (1979), De la séduction, Paris, Galilée.

BOËTSCH, G., GUILHEM, D. (2005), « Rituels de séduction », Hermès, La Revue, vol. $3, \mathrm{n}^{\circ} 43$, pp. 179-188.

CARDON, D. (2010), La démocratie Internet. Promesses et limites, Paris, Seuil.

CASILLI, A. A. (2010), Les liaisons numériques. Vers une nouvelle sociabilité ?, Paris, Seuil.

CERVUlle, M., PAILlER, F. (2014), «\#mariagepourtous : Twitter et la politique affective des hashtags ", Revue française des sciences de l'information et de la communication, $\mathrm{n}^{\circ} 4$.

CHARRON, J. (1994), La production de l'actualité. Une analyse stratégique des relations entre la presse parlementaire et les autorités politiques au Québec, Montréal, Boréal.

CHARRON, J. (1998), « Les parlementaires et les médias : un mariage d'intérêt ? », Le Parlementarisme et les médias, Actes du colloque, Hôtel du Parlement, Québec, pp. 159-161.

CHIBOIS, J. (2014), «Un “appeau à journaliste” pour les députés, ou Twitter comme antichambre à la publicité parlementaire », in Zlitni, S., Liénard, F., Dula, D., Crumière, C. (dir.), Actes du colloque international Communication Électronique, Cultures et Identités, Le Havre, Klog Éditions, pp. 371-378.

CHIBOIS, J. (11 octobre 2013), « Twittographie parlementaire (4). Trombinoscope », LASPIC | Carnet [En ligne]. URL : http://laspic.hypotheses.org/1789.

DELPORTE, C. (2012), Une histoire de la séduction politique, Paris, Flammarion.

DUPONT, C. (1982), La négociation. Conduite, théorie, applications, Paris, Dalloz.

GARRIGUES, J. (2007), Histoire du parlement de 1789 à nos jours, Paris, Colin.

GINGRAS, A.-M. (2009), Médias et démocratie : le grand malentendu, Québec, Presses de l'Université du Québec.

HOURQUEBIE, F. (2013), « Chaînes parlementaires \& séparation des pouvoirs », in Touzeil-Divina, M. (dir.), Le Parlement aux écrans !, Le Mans, Éditions L’Épitoge, pp. 13-21.

JACKSON, N., LILlEKER, D. (2011), « Microblogging, Constituency Service and Impression Management: UK MPs and the Use of Twitter », The Journal of Legislative Studies, vol. 17, $\mathrm{n}^{\circ} 1$, pp. 86-105. 
KERROUCHE, É. (2009), «Usages et usagers de la permanence du député », Revue française de science politique, vol. 59, $\mathrm{n}^{\circ} 3$, pp. 429-454.

LAVOINNE, Y. (1999), « Publicité des débats et espace public », in Études de communication. Langages, information, médiations, $\mathrm{n}^{\circ} 22$, pp. 115-132.

LEMIEUX, C. (2000), Mauvaise presse: une sociologie compréhensive du travail journalistique et de ses critiques, Paris, Métailié.

LESTON-BANDEIRA, C., BENDER, D. (2013), « How Deeply are Parliaments Engaging on Social Media », Information Polity, vol. 18, n 4, pp. 281-297.

LE TORREC, V. (2005), « Aux frontières de la publicité parlementaire : les assemblées et leur visibilité médiatisée », Réseaux, n 129-130, vol. 1, pp. 181-208.

MARTIN, M. (1997), Médias et journalistes de la République, Paris, Odile Jacob.

MERZEAU, L. (2013), "Twitter : une machine à fabriquer de l'autorité », in Gallezot, G., Pélissier, N. (dir.), Twitter. Un monde en tout petit ?, Paris, L'Harmattan, pp. 35-51.

PÉLISSIER, N., DIALLO, M. D. (2013), « Le journalisme est-il soluble dans Twitter? Enquête sur le choc de deux médiacultures », in Gallezot, G., Pélissier, N. (dir), Twitter. Un monde en tout petit?, Paris, L'Harmattan, 2013, pp. 163-178.

PÉLISSIER, N., GALLEZOT, G. (2013), « Twitter : un micro-monde sans fin(s) ? », in Gallezot, G., Pélissier, N. (dir), Twitter. Un monde en tout petit?, Paris, L'Harmattan, pp. 15-31.

ROGINSKY, S., JEANNE-PERRIER, V. (2013), « La fabrique de la communication des parlementaires européens : "Tweet ton député" et les "ateliers du député 2.0" ", Politiques de communication, $\mathrm{n}^{\circ} 3$, pp. 163-178, 85-123.

THOMPSON, J.-B. (2005), « La nouvelle visibilité », Réseaux, n 129-130, vol. 1, pp. 59-87.

URVOAS, J.-J., ALEXANDRE, M. (2012), Manuel de survie à l'Assemblée nationale. L'art de la guérilla parlementaire, Paris, Odile Jacob.

VOIROL, O. (2005), « Les luttes pour la visibilité. Esquisse d'une problématique », Réseaux, $\mathrm{n}^{\circ} 129-130$, vol. 1, pp. 89-121.

\section{Articles de presse}

AFP (2010), «L'Assemblée se penchera sur l'usage que ses membres font de Twitter », LeMonde.fr [En ligne], 2 juillet, URL : http://www.lemonde.fr/imprimer/ article/2010/07/02/1381934.html (consulté le 3 février 2013).

BEKMEZIAN H. (2013), « Au grand marché de l'Assemblée », Le Monde, 21 septembre. 
DRYEF Z. (2012), « Moi, député, je veux percer dans les médias », Rue89 [En ligne], 22 décembre. URL : http://www.rue89.com/rue89-politique/2012/12/22/moi-deputeje-veux-percer-dans-les-medias-238013 (consulté le 21 janvier 2013).

HAGUET H. (2012), « 4 colonnes, 577 députés, 300 mètres carrés ». L'Express.fr [En ligne], 11 juillet. URL : http://www.lexpress.fr/actualite/politique/4-colonnes-577deputes-300-metres-carres_1136994.html (consulté le 24 mai 2014).

HUET S. (2011), «Aux “Quatre Colonnes”, les députés sous l'œil des médias », Le Figaro, 9 août. 